\title{
Mutants in the Fe-S biogenesis component AtDRE2 develop twin embryos and are defective in DNA demethylation in the vegetative phase
}

\author{
Diana Mihaela Buzas ${ }^{\star 1}$, Tetsu Kinoshita ${ }^{2}$ \\ ${ }^{1}$ Life and Environmental Sciences and Gene Research Center, University of Tsukuba 1-1-1 Tennodai, Tsukuba, \\ Ibaraki 305-8571, Japan \\ ${ }^{2}$ Kihara Institute for Biological Research, Yokohama City University, 641-12 Maioka Totsuka, \\ Yokohama, Kanagawa, 244-0813, Japan
}

(Received April 6, 2018; accepted 7 June 2018)

\begin{abstract}
Iron is assembled inside cells into cofactors, essential for biological functions. Two types of cofactors, Iron Sulfur clusters and diferric tyrosyl radicals, share a conserved assembly complex containing the DRE2 enzyme, essential for cell viability in eukaryotes. In the model plant Arabidopsis thaliana, dre 2 mutants are lethal, having a maternal defect related to active DNA demethylation in the central cell gamete and a zygotic defect leading to embryo arrest. However, neither of these defects is fully penetrant. We were able to recover the first dre 2 viable mutants ever reported in plants by expressing DRE2 from a central cell specific promoter in a dre 2 heterozygote background. The viable dre 2 mutants developed twin embryos infrequently and were defective in DNA demethylation in the vegetative phase. These mutants represent a valuable tool to uncover further processes dependent on Iron containing cofactors from plants that may also be universal.
\end{abstract}

Keywordss plant reproduction, DNA methylation, Iron Sulfur biogenesis

\section{INTRODUCTION}

Iron cofactors are essential for many cellular processes, frequently participating in electron transfer, catalytic reactions and gene regulation (1). Iron-Sulfur (Fe-S) clusters and the diferric tyrosyl radical cofactor are two types of Iron cofactors assembled by dedicated cellular machineries. In eukaryotes, Fe-S biogenesis is compartmentalized in organelles via pathways of prokaryotic origin and in the cytosol, via the CIA (Cytosolic Iron Sulfur Assembly) pathway. The CIA proteins are well-characterized in yeast and belong to three functional classes: 1. Electron transfer chain, composed of the diflavin reductase Tah18 (Top1T722A mutant Hypersensitive 18) and the $\mathrm{Fe}-\mathrm{S}$ protein Dre2 (Derepressed for Ribosomal protein S14 Expression 2) (2);
2. Fe-S scaffold proteins including Npb35 (nucleotide binding protein 35) and Cdf1 (cytosolic Fe-S cluster deficient 1) (3); 3. Fe-S targeting complex proteins composed of four proteins: Nar1 (Nuclear architecture related 1), Cia1, Cia2, and Met18 (4). CIA pathway is responsible for the maturation of enzymes in the cytosol and in the nucleus. Some of these are known - for example, many DNA metabolism enzymes are Fe-S dependent (5) - but currently, a sizeable fraction of Fe-S proteins are unidentified (6). In addition to the CIA pathway, Tah18-Dre2 complex was recently found to function in diferric-tyrosyl radical formation, a cofactor required for the ribonucleotide reductase $(7,8)$.

Although CIA proteins are highly conserved in eukaryotes, it has been difficult to reveal what are the cellular processes controlled by $\mathrm{Fe}-\mathrm{S}$ clusters because

*Corresponding author:

Diana Mihaela Buzas, PhD, Associate Professor,

University of Tsukuba, Faculty of Life and Environmental Sciences Room B715,

Gene Research Centre, Room 218, 1-1-1 Tennodai Tsukuba, Ibaraki, 305-8572 Japan

Tel.: +81 0298536685, 0298536446;

Email: buzas.mihaela.ka@u.tsukuba.ac.jp 
the CIA components are often lethal when mutated. In plants, mainly Arabidopsis thaliana (At), CIA mutants are embryo lethal, except MET18 (9-11). Few strategies were employed so far to overcome lethality of orthologous CIA proteins with scaffold and targeting function. For example, a role of AtNBP35 in establishing leaf morphology could be inferred from combining know-down lines with an amino acid substitution in AtNBP35 (12). Also, a weak allele in AtNAR1 revealed its function in oxidative stress responses in vegetative cells (13) while a weak allele of AE7 (ASYMETRIC LEAVES1/2 ENHANCER 7), the putative $\mathrm{Cia} 2$ orthologue in plants, uncovered a role in nuclear genome integrity via a CIA function and in leaf polarity (14). However, so far, viable homozygote alleles in early electron transfer CIA proteins have not been reported in plants.

Unique links between CIA proteins and a family of Fe-S dependent DNA glycosylases specialized in DNA demethylation, composed of DEMETER (DME), ROS1 (REPRESSOR OF SILENCING 1), DML2 and 3 (DEMETER-like 2 and 3), were uncovered in both the reproductive and the vegetative phases in Arabidopsis. DME targets genes for DNA demethylation coupled with transcriptional activation in the central cell maternal gamete, including one termed FWA (FLOWERING WANGENINGEN). A $p F W A ; G F P$ reporter can easily monitor DME activity and was used in a genetic screen which allowed isolation of heterozygote EMS alleles of AtDRE2 (10) and AtNAR1 (13). Further evidence highlighted that dme and dre2, but not the other CIA mutants, share maternal phenotypic hallmarks (10), indicating a distinctive function for AtDRE2 in the maternal central cell gamete. Additionally, like all CIA proteins, AtDRE2 has a zygotic role in early embryo development $(9,10)$. The other three demethylases, ROS1, DML2 and 3, are active in vegetative cells (15). Two other CIA proteins, AtAE7 and AtMET18, were also implicated in DNA demethylation in vegetative cells $(11,14)$.

The epigenetic role of AtDRE2 could be revealed using a heterozygote allele due to its maternal gamethophytic function (10). However, because AtDRE2 has potential to regulate a myriad of other processes outside gametes via its putative role in $\mathrm{Fe}-\mathrm{S}$ cluster biogenesis and diferric-tyrosyl radical formation, it is of interest to also obtain viable tissues where both alleles are mutated, i.e., homozygote dre2. We succeeded in recovering viable dre 2 homozygotes by complementing the DRE2 female gamethophytic function. Here we report on the initial characterization of new phenotypes in the reproductive phase and on defects in DNA demethylation in vegetative cells in these mutants.

\section{MATERIALS AND METHODS}

Plant growth, transgenic construction and genotyping. The Arabidopsis thaliana lines used in this study are WT Col-0, dre2-2 Col-0 - the EMS allele generated as described in Buzas et al (2014) (10) - and triple mutant $r d d$ (ros 1-3; dml2-1;dml3-1) Col, that was generated by Penterman et al (2007) (15). Seeds were sterilized, sowed on MS plates with 3\% sucrose, stratified for three days, then grown for a further two weeks on plates before transferring to pots in a two parts vermiculite: one part soil mixture. Plants were cultivated under standard long days light conditions for Arabidopsis.

Three independent complementation lines in dre2-2 were obtained with a cassette containing a central cell specific promoter of At2g24840 (named DIANA gene) constructed as described in Buzas et al (2014), and one line was analysed in detail. For dre2-2 genotyping, genomic DNA was amplified with GGCAAAGAAACCTTCTTGGAA and TGGGGGTTGAGTTTAGTTGG oligonucleotides and products were digested with HindIII before electrophoresis.

Histology and microscopy. For whole-mount histology, different stage siliques and were dissected manually and then cleared on a microscope slide under the glass cover in a mixture of chloral hydrate, glycerol, and water $(8 \mathrm{~g}: 1 \mathrm{~mL}: 2 \mathrm{~mL})$ for at least four hours at room temperature. Fluorescence images were captured using a Zeiss Axioimager M1 microscope equipped with a Zeiss AxioCam MRc 5 and Nomarski optics.

Bisulphite genomic DNA sequencing and analysis. DNA was extracted using a CTAB method from different genotypes at 12 days after germination stage and was subjected to the bisulfite reaction using the Epitect bisulfite Kit (Qiagen, \#59104). The ASA1 gene was used as a positive control for the bisulfite conversion as described by Ikeda et al (2011) (16). The oligonucleotide sequences for At4g14365, At1g53860 and Atlg26400 were as in (15). Sequence alignments and trimming were done using: SegManPro under DNAStar software, web based clustalw tools and CyMATE (17).

\section{RESULTS}

\section{Isolation of viable dre2-2}

We previously demonstrated that expression of a AtDRE2 transgene driven by a central cell specific promoter from DIANA gene can restore the defect in activation of $p F W A$; GFP in dre2-2 +/- both in the central cell and the endosperm, but not the other zygotic mutant seed phenotypes (10). We hypothesized that: i) the expression of other AtDRE2-regulated genes important for viability may be similarly restored in the endosperm lineage; and ii) because the mutant seed phenotype is not fully penetrant in the embryonic lineage $(10,18)$, we may be able to recover dre2-2 homozygote progeny once 
Table 1. Seed genotype of different phenotypic classes from a homozygote transgenic line expressing AtDRE2 in the central cell in dre2-2+/- background

\begin{tabular}{|l|l|l|l|l|l|}
\multicolumn{1}{c}{$\begin{array}{c}\text { Seed } \\
\text { phenotype }\end{array}$} & \multicolumn{2}{c}{$\begin{array}{c}\text { Genotype } \\
\text { dre2-2+/- }\end{array}$} & \multicolumn{2}{c|}{ dre2-2 } & Total seed $\begin{array}{c}\text { \% Observed } \\
\text { homozygote }\end{array}$ \\
\hline Distorted & 0 & 1 & 20 & 21 & $95.2 \%$ \\
\hline Random & 11 & 25 & 2 & 38 & $5.2 \%$ \\
\hline
\end{tabular}

the gamethophytic defect is restored. We first proceeded to close examination of dry seed of a pDIANA:DRE2; dre2-2+/- progeny. Using a stereomicroscope, we found a class of dry mutant seed, unseen in wild type or in any other AtDRE2 transgenic constructs, at a low frequency: some seed appeared distorted towards the mycropylar/chalazal end (Fig. $1 \mathrm{~A}-\mathrm{C}$ ). This seemed to be caused by a distortion in embryo shape from the hypocotyl area (Fig. 1 D, E). We then selected dry seed with distorted phenotype under a stereomicroscope and sowed them. All seeds germinated and were genotyped for dre2-2 allele. We
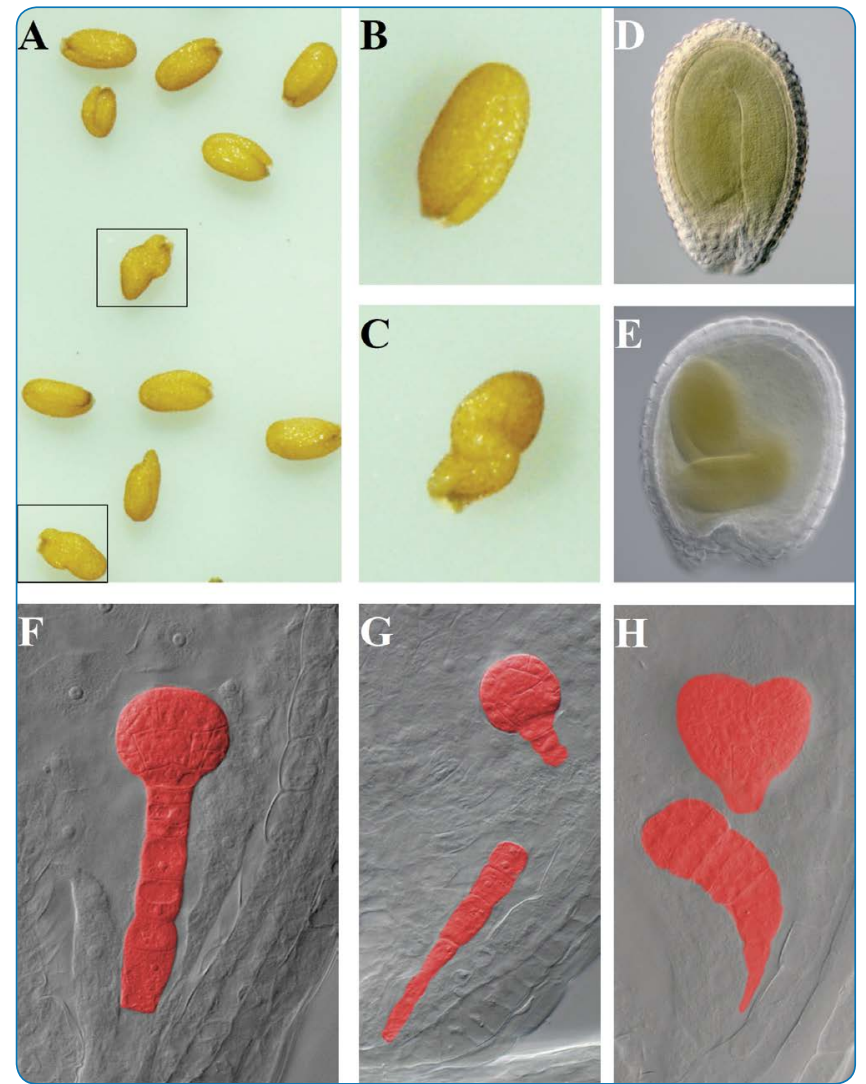

Fig. 1. Isolation of viable dre2-2 mutants and the twin embryo phenotype in the first generation. A. Seed progeny from pDIANA:DRE2;dre2-2+/- self-pollinated plants contained wild type looking seed as well as some distorted seed (black rectangulars); B and C. Examples of phenotypic classes of wild type and distorted seed, respectively; D and E. Nomarski images of cleared wild type and seed distorted seed, respectively; F-H. Representative images of embryos at globular and heart stage with suspensor from a pDIANA:DRE2;dre2-2-/- sillique; F. Wild type phenotype; and $\mathbf{G}, \mathbf{H}$. Twin embryo phenotype. found that $95.2 \%$ (20 out of 21 ) of seeds with distorted phenotype were dre2-2 homozygote. Therein, we refer to pDIANA:DRE2; dre2-2 as "viable dre2-2". We also genotyped random seed from the same progeny. In this case, only $5.2 \%$ (2 out of 38 ) homozygote were identified (Table 1). In conclusion, we were able to recover viable dre2-2 at a low frequency, as expected.

Mutant phenotypes of viable dre2-2 during reproductive development

To obtain information on reproductive development, we dissected siliques in different stages of development from dre2-2 viable plants. We noticed that only a proportion of ovules developed into seeds in late stages $(68 \%, \mathrm{n}=534)$, indicating that dre2-2 viable has reduced fertility. This phenotype was not due to AtDRE2 expression from pDIANA;DRE2 transgene because all ovules developed normally in transgenic lines without the dre2-2 allele.

While examining seeds from early globular to heart stage, we found a rare phenotype: a second embryo developed in vicinity of the main embryo, possibly from cells of the suspensor. This phenotype appeared at a low frequency of approximately 1:500 (Fig. 1 F-H).

Therefore, we found at least two additional reproductive phenotypes of the dre 2 viable, not seen in the heterozygote background: reduced fertility and twin embryos.

Viable dre2-2 has DNA demethylation defects in vegetative phase

In the vegetative phase, the growth of seedlings produced from germinating distorted seed was slower than wild type in the first two weeks on Petri dishes, but we found that they can recover to normal growth in soil up to the flowering stage.

It is known that $A t D R E 2$ reduces the DNA methylation levels in the endosperm linage at genes targeted by DEMETER mediated demethylation (10). Now that we were able to obtain a viable dre2-2, we asked if genes targeted by DNA demethylation in vegetative tissues are also affected. We chose three genes from those reported to be targeted by DNA demethylases in vegetative tissues: At1g53860 and At4g14365, where three demethylases act redundantly and At1g26400, where only ROS1 demethylates (15). We quantified and visualized DNA methylation in the three sequence contexts using CYMATE (17) (Fig. 2). 
At Atlg53860 and At4g14365, DNA methylation increased in all contexts in $r d d$ relative to wild type, mutant as shown before (17). The same was also true for dre2-2 viable mutant, although the differences tended to be weaker. A detailed look at the specific residues that undergo DNA demethylation, for example in CG context, indicated several classes of differential CG methylation in the three genotypes. Notably, some CGs were hypermethylated in both mutants (e.g., C135, C140, C210 in At4g14365; C42, C47, C55, C71 in At1g53860; see gray shading in Fig. $2 \mathrm{~A}, \mathrm{~B}$ ). There also appear to be CGs where methylation resides specifically in one mutant genotype (e.g., in dre2 viable only: C30 in At4g14365; C336, C338 in At1g53860; see purple shading in Fig. 2 A, B; in rdd only: C162, C177 in At1g53860; see green shading in Fig. $2 \mathrm{~A}, \mathrm{~B})$.

At At1g26400, the methylation pattern was more complex (Fig. 2 C). In addition to the aforementioned hypermethylation classes at common (C60, C256 in At1g26400; gray shading, Fig. 2 C) and specific (C22 in dre2 viable only, purple shading, Fig. 2 C; C181, C195 and $\mathrm{C} 204$ in $r d d$, green shading, Fig. 2 C) residues, DNA methylation decreased at one common CG in both mutants (C105; purple and green boxes, Fig. 2 C) or in specific locations in each mutant (C204, C226, C243 in viable; C22, $\mathrm{C} 118$ in $r d d$, purple and green boxes, Fig. 2 C).

Altogether, we conclude that viable dre2-2 mutants shared defects in the pattern of DNA methylation with $r d d$ mutants in vegetative phase, indicative of a role for AtDRE2 in DNA demethylation in these tissues.

\section{DISSCUSSION}

DRE2 is a conserved eukaryotic Fe-S cluster biogenesis proteins, best known for its function in the cytosolic Fe-S biogenesis pathway (19). Since the initial report in yeast

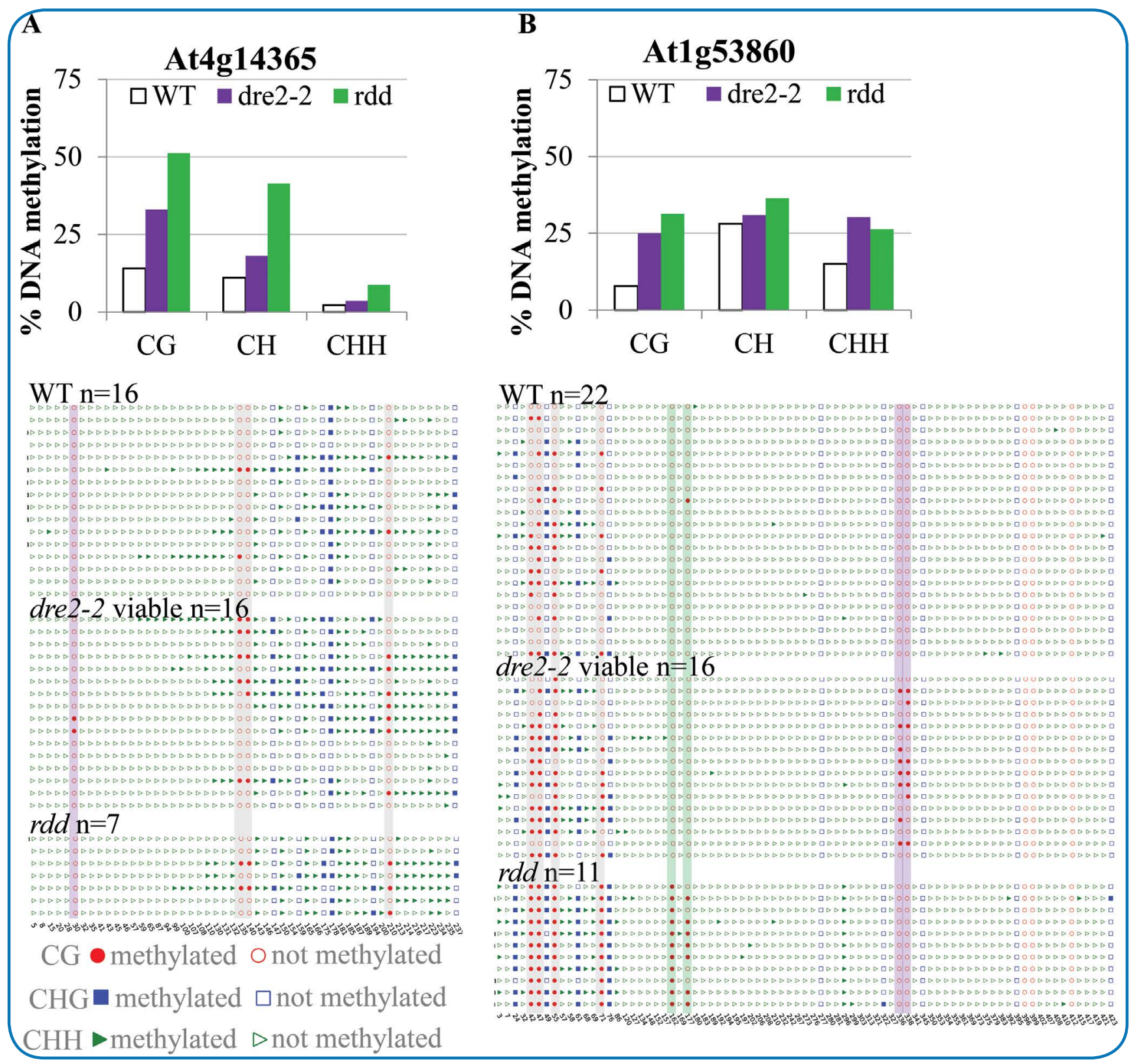




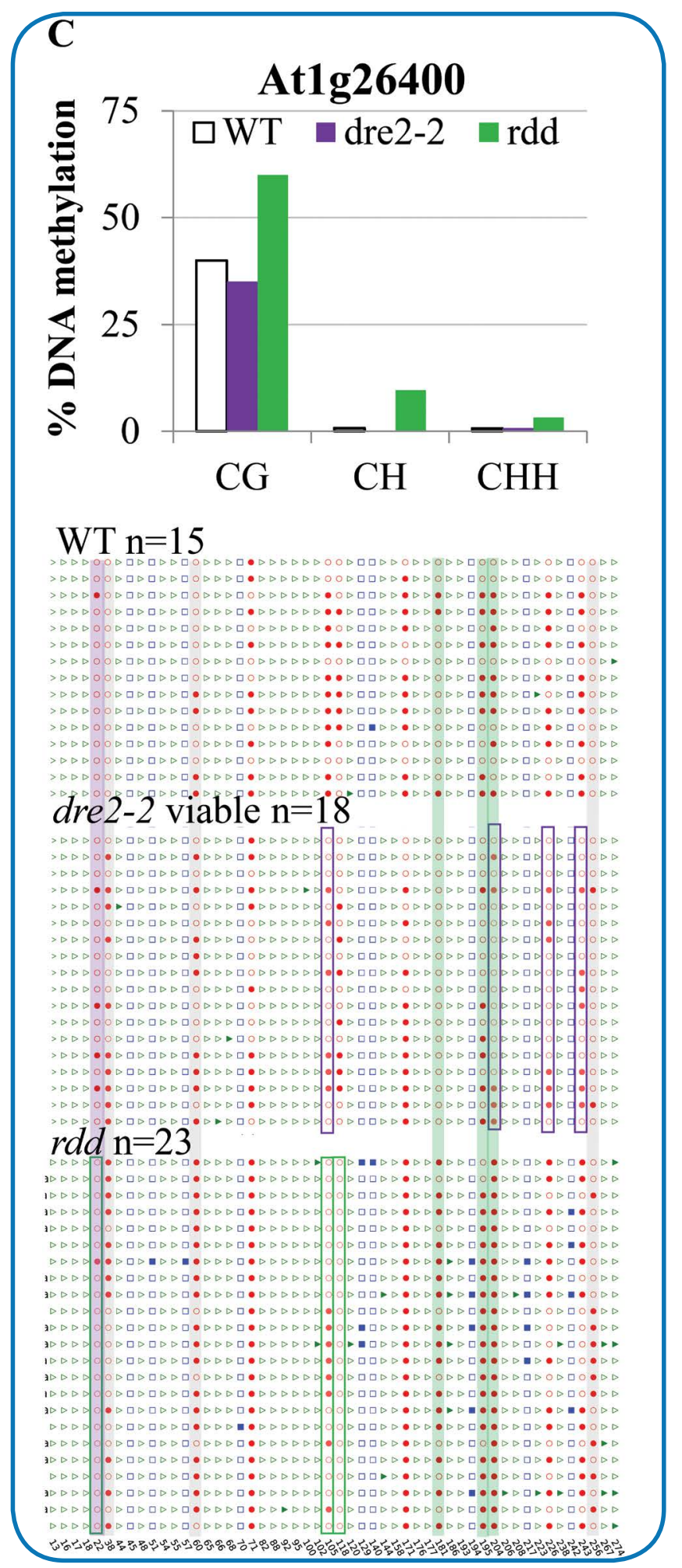

(20), a number of biological roles have been associated with DRE2. For example, in mice it is an antiapoptotic molecule essential for definitive hematopoiesis (21) and in plants it has an epigenetic role related to DNA demethylation in one maternal gamete $(10,18)$. However, based on its known biochemical functions $(2,7)$ DRE2 likely impacts more biological processes than currently acknowledged.

AtDRE2 is expressed broadly in Arabidopsis thaliana. Notable AtDRE2 expression is in structures which participate dynamically in fertilization and seed
Fig. 2. DNA methylation in vegetative tissue of WT, dre2-2 viable and $r d d$ plants at three demethylation genes. CYMATE quantification (top panels) and DNA methylation profile (lower panels) for At4g14365 (A), Atlg53860 (B) and Atlg26400 (C).

The sequences are located between +2493 to +2743 from ATG for At1g53860 (situated downstream of the 3'UTR of the gene); +1898 to +2323 from ATG for At4g14365 (situated over the $3^{\prime}$ UTR of the gene) and +1193 to +1470 from ATG for Atlg26400 (situated within the coding region). Number of clones sequenced for each genotype is indicated by "n". Red circle indicates a CG residue, blue square indicates a $\mathrm{CHG}$ residue and green triangle indicates a $\mathrm{CHH}$ residue, all of which are either methylated (full) or nonmethylated (contoured). The numbers under the CYMATE images represent locations of cytosines within the reference sequence amplicon. Shading indicates specific CG hypermethylation and boxes indicate specific hypomethylation, where differences to wild type were higher than $20 \%$ : gray, both mutants, purple viable dre2-2 only, green $r d d$ only. 
during DRE complementation of central cell function, the events leading to dre2 viable homozygote recovery had a low frequency. These events probably took place in seeds where the dre 2 embryo phenotype is weak, leading to distorted, yet viable, seed. In a very small proportion of viable seeds, two embryos formed. This is an interesting, unexpected phenotype. Twin embryos can form in many taxa in higher plants yet always infrequently. In Arabidopsis, some suspensor cells in twin mutant develop into embryos (22). It remains possible that viable dre2 also initiate embryos from suspensor cells because twin embryos were always located in the vicinity of suspensor cells. It was, however, difficult to investigate this in detail due to the very low frequency of this phenotype.

The AtDRE2 roles during reproductive development may be exerted via a critical $\mathrm{Fe}-\mathrm{S}$ protein, as we previously demonstrated that $D M E$ is a strong candidate $\mathrm{Fe}-\mathrm{S}$ protein for maternal function $(10,18)$. However, more detailed analysis in the tiny central cell embedded in a mass of sporophytic tissue remains difficult. In addition to the AtDRE2 expression during reproductive phase, it is also present in vegetative cells (10). To understand if the activity of the Fe-S-dependent DNA glycosylates active in vegetative cells (ROS1, DML2 and DML3) is also reduced when AtDRE2 function is abolished, we analyzed DNA methylation levels at three target demethylation genes in our viable dre2 mutant. An increase in DNA methylation levels was especially clear in the CG context in the dre 2 viable comparing to the wild type at two out of the three genes investigated. We found that cytosine residues hypermethylated in rdd mutant can also be hypermethylated in the viable dre 2 mutant (see gray shading, Fig. 2). These results indicate that AtDRE2 is required for activity of the DNA demethylase operating in vegetative cells, as is the case for the central cell maternal gamete. The DNA methylation changes was deeper in $r d d$ than in viable $d r e 2$ at most (7 out of 10) common hypermethylated cytosines. It is possible that AtDRE2 is not absolutely required for DNA demethylation, based on redundancy, or that AtDRE2 influences processes of both DNA methylation and de-methylation.

In conclusion, here we generated a genetic tool enabling us to uncover novel phenotypes associated with AtDRE2 function. This tool from plants will also facilitate further future investigations at the molecular level towards expanding our understanding of the biological processes dependent on iron containing cofactors, that may be common to all eukaryotes.

Author contributions: D.B. designed the experiments, conducted the research and wrote the manuscript; T.K. contributed materials and commented on the manuscript.

Acknowledgments: We acknowledge excellent technical assistance from Yuki Kinoshita with bisulphite sequence analysis and Yukiko Sugimoto with plant growth and genotyping. Adobe work in Fig. 1 F-H was done by Laura Buzas and Petre Nicolescu. This work was supported by the Ministry of Education, Culture, Sports, Science and Technology, Japan: Grant-in-Aid for Young Scientists B grant No. $15 \mathrm{~K} 18558$ to D.B., Scientific Research (B) grant No. 17H03749 to T.K., and Scientific Research on Innovative Areas, grant No. $16 \mathrm{H} 01459$ to D. B. and grant No. $16 \mathrm{H} 06471$ to T.K.

\section{REFERENCES}

1. Lill R, Function and biogenesis of iron-sulphur proteins, Nature, 460, 831-838 (2009).

2. Netz DJA, StümpFigure M, Doré C, Mühlenhoff U, Pierik AJ, Lill R, Tah18 transfers electrons to Dre2 in cytosolic iron-sulfur protein biogenesis, Nat. Chem. Biol., 10, 758-765 (2010).

3. Netz DJA, Stith CM, StümpFigure M, Köpf G, Vogel D, Genau HM, et al, Eukaryotic DNA polymerases require an iron-sulfur cluster for the formation of active complexes, |Nat. Chem. Biol., 1, 125-132 (2012).

4. Netz DJA, Mascarenhas J, Stehling O, Pierik AJ, Lill R, Maturation of cytosolic and nuclear iron-sulfur proteins, Trends in Cell Biol., 24, 303-312 (2014).

5. White MF, Dillingham MS. Iron-sulphur clusters in nucleic acid processing enzymes. Curr. Op. Struct. Biol., 22, 94-100 (2012).

6. Rouault TA, Iron-sulfur proteins hiding in plain sight, Nat. Chem. Biol., 11, $442-445$ (2015).

7. Zhang Y, Li H, Zhang C, An X, Liu L, Stubbe J, et al, Conserved electron donor complex Dre2-Tah18 is required for ribonucleotide reductase metallocofactor assembly and DNA synthesis,

Proc. Natl. Acad. Sci. U.S.A., 17, E1695-E1704 (2014).

8. Li H, StümpFigure M, Zhang C, An X, Stubbe JA, Lill R, et al,
The diferric-tyrosyl radical cluster of ribonucleotide reductase and cytosolic iron-sulfur clusters have distinct and similar biogenesis requirements, J. Biol. Chem., 27, 11445-1151(2017).

9. Bernard DG, Netz DJA, Lagny TJ, Pierik AJ, Balk J, Requirements of the cytosolic iron-sulfur cluster assembly pathway in Arabidopsis, Philos. Trans. R. Soc. B. Biol. Sci., 1622, 20120259-20120259 (2013).

10. Buzas DM, Nakamura M, Kinoshita T, Epigenetic role for the conserved Fe-S cluster biogenesis protein AtDRE2 in Arabidopsis thaliana, Proc. Natl. Acad. Sci. U.S.A., 37, 13565-13570 (2014).

11. Duan CG, Wang X, Tang K, Zhang H, Mangrauthia SK, Lei M, et al, MET18 Connects the Cytosolic Iron-Sulfur Cluster Assembly Pathway to Active DNA Demethylation in Arabidopsis,

PLoS. Genet., 11, e1005559 (2015).

12. Bastow EL, Bych K, Crack JC, Le Brun NE, Balk J, NBP35 interacts with DRE2 in the maturation of cytosolic iron-sulphur proteins in Arabidopsis thaliana, Plant J., 3, 590-600 (2017).

13. Nakamura M, Buzas DM, Kato A, Fujita M, Kurata N, Kinoshita $\mathrm{T}$, The role of Arabidopsis thaliana NAR1, a cytosolic iron-sulfur cluster assembly component, in gametophytic gene 
expression and oxidative stress responses in vegetative tissue, New Phytol., 2, 925-935 (2013).

14. Luo D, Bernard DG, Balk J, Hai H, Cui X, The DUF59 Family Gene AE7 Acts in the Cytosolic Iron-Sulfur Cluster Assembly Pathway to Maintain Nuclear Genome Integrity in Arabidopsis, Plant Cell, 24, 4135-4148 (2012).

15. Penterman J, Zilberman D, Huh JH, Ballinger T, Henikoff S, Fischer RL, DNA demethylation in the Arabidopsis genome, Proc. Natl. Acad. Sci. U.S.A., 16, 6752-6757 (2007).

16. Ikeda Y, Kinoshita Y, Susaki D, Ikeda Y, Iwano M, Takayama S, et al, HMG Domain containing SSRP1 is required for DNA demethylation and genomic imprinting in arabidopsis, Dev. Cell., 3, 589-596 (2011).

17. Hetzl J, Foerster AM, Raidl G, Scheid OM, CyMATE: A new tool for methylation analysis of plant genomic DNA after bisulphite sequencing, Plant J., 3, 526-536 (2007).

18. Buzas DM, Emerging links between iron-sulfur clusters and 5-methylcytosine base excision repair in plants,

Genes Genet. Syst., 2, 51-62 (2016).

19. Zhang Y, Lyver ER, Nakamaru-Ogiso E, Yoon H, Amutha B, Lee D-W, et al, Dre2, a Conserved Eukaryotic Fe/S Cluster Protein, Functions in Cytosolic Fe/S Protein Biogenesis, Mol. Cell. Biol., 18, 5569-582 (2008).

20. Chanet R, Heude M. Characterization of mutations that are synthetic lethal with pol3-13, a mutated allele of DNA polymerase delta in Saccharomyces cerevisiae, Curr. Genet., 5, 337-350 (2003).

21. Shibayama H, Takai E, Matsumura I, Kouno M, Morii E, Kitamura Y, et al, Identification of a Cytokine-induced Antiapoptotic Molecule Anamorsin Essential for Definitive Hematopoiesis, J. Exp. Med., 4, 581-592 (2004).

22. Vermon DM, Meinke DW, Embryogenic transformation of the suspensor in twin, a polyembryonic mutant of Arabidopsis, Dev. Biol., 2, 566-573 (1994). 